\title{
Cloud Atlas: Rotational Spectral Modulations and Potential Sulfide Clouds in the Planetary-mass, Late T-type Companion Ross 458C
}

\author{
Elena Manjavacas ${ }^{1,2}$ (10), Dániel Apai ${ }^{2,3,12}$ (1), Ben W. P. Lew ${ }^{3}$ (1), Yifan Zhou ${ }^{2}$ (1) , Glenn Schneider ${ }^{2}$ (10), Adam J. Burgasser ${ }^{4}$ (D), \\ Theodora Karalidi ${ }^{5}$ (i), Paulo A. Miles-Páez ${ }^{2,6}$ (i) , Patrick J. Lowrance ${ }^{7}$, , Nicolas Cowan ${ }^{8}$ (i), Luigi R. Bedin ${ }^{9}$, Mark S. Marley ${ }^{10}$ (i), \\ Stan Metchev ${ }^{6}$ (i), and Jacqueline Radigan ${ }^{11}$ \\ ${ }^{1}$ W.M. Keck Observatory, 65-1120 Mamalahoa Highway, Kamuela, HI 96743, USA; emanjavacas@keck.hawaii.edu \\ ${ }^{2}$ Department of Astronomy/Steward Observatory, The University of Arizona, 933 N. Cherry Avenue, Tucson, AZ 85721, USA \\ ${ }^{3}$ Department of Planetary Science/Lunar and Planetary Laboratory, The University of Arizona, 1640 E. University Boulevard, Tucson, AZ 85718, USA \\ ${ }^{4}$ Center for Astrophysics and Space Science, University of California San Diego, La Jolla, CA 92093, USA \\ ${ }^{5}$ Department of Astronomy and Astrophysics, University of California, Santa Cruz, California, USA \\ ${ }^{6}$ The University of Western Ontario, Department of Physics and Astronomy, 1151 Richmond Avenue, London, ON N6A 3K7, Canada \\ ${ }_{7}^{7}$ IPAC-Spitzer, MC 314-6, California Institute of Technology, Pasadena, CA 91125, USA \\ ${ }^{8}$ Department of Earth \& Planetary Sciences, 3450 University St. Montreal, Quebec H3A 0E8, Canada \\ INAF-Osservatorio Astronomico di Padova, Vicolo Osservatorio 5, I-35122 Padova, Italy \\ ${ }^{10}$ NASA Ames Research Center, Mail Stop 245-3, Moffett Field, CA 94035, USA \\ ${ }^{11}$ Utah Valley University, 800 West University Parkway, Orem, UT 84058, USA \\ Received 2019 January 29; revised 2019 March 25; accepted 2019 March 25; published 2019 April 16
}

\begin{abstract}
Measurements of photometric variability at different wavelengths provide insights into the vertical cloud structure of brown dwarfs and planetary-mass objects. In seven Hubble Space Telescope consecutive orbits, spanning $\sim 10 \mathrm{hr}$ of observing time, we obtained time-resolved spectroscopy of the planetary-mass T8 dwarf Ross $458 \mathrm{C}$ using the near-infrared Wide Field Camera 3. We found spectrophotometric variability with a peak-to-peak signal of $2.62 \pm 0.02 \%$ (in the $1.10-1.60 \mu \mathrm{m}$ white light curve). Using three different methods, we estimated a rotational period of $6.75 \pm 1.58 \mathrm{hr}$ for the white light curve, and similar periods for narrow $J$ - and $H$-band light curves. Sine wave fits to the narrow $J$ - and $H$-band light curves suggest a tentative phase shift between the light curves with wavelength when we allow different periods between both light curves. If confirmed, this phase shift may be similar to the phase shift detected earlier for the T6.5 spectral type 2MASS J22282889-310262. We find that, in contrast with $2 \mathrm{M} 2228$, the variability of Ross $458 \mathrm{C}$ shows evidence for a color trend within the narrow $J$-band, but gray variations in the narrow $H$-band. The spectral time-resolved variability of Ross $458 \mathrm{C}$ might be potentially due to heterogeneous sulfide clouds in the atmosphere of the object. Our discovery extends the study of spectral modulations of condensate clouds to the coolest T-dwarf, planetary-mass companions.
\end{abstract}

Key words: brown dwarfs - stars: atmospheres

\section{Introduction}

At effective temperatures close to $1400 \mathrm{~K}$-within a relatively narrow range-brown dwarf upper atmospheres undergo a radical transformation that marks the transition between the $\mathrm{L}$ and $\mathrm{T}$ spectral classes. In the near-infrared spectra of $\mathrm{T}$ dwarfs, methane absorption features appear and near-infrared colors shift dramatically to bluer (Burgasser et al. 2002a; Cushing et al. 2005; Kirkpatrick 2005). Below this temperature, silicate and iron clouds seem to play a less important role in shaping the emerging spectra than for L-type dwarfs. This transition is thought to be due to clouds sinking below the photosphere (Ackerman \& Marley 2001; Burgasser et al. 2002b).

Time-resolved photometry and spectroscopy allow isolating changes in cloud properties from the bulk properties of the objects. Models of spectrophotometric variability in $\mathrm{T}$ dwarfs like the T2 SIMPJ013656.5+093347 (SIMP0136; Artigau et al. 2009; Apai et al. 2013), the T2.5 2MASS J213926760220226 (2M2139; Radigan et al. 2012; Apai et al. 2013), and the T6.5 2MASS J22282889-4310262 (2M2228; Buenzli et al.

2012) show that cloud layers of varying thicknesses are present in the atmospheres of $\mathrm{T}$ dwarfs. Extensive monitoring

\footnotetext{
12 Earths in Other Solar Systems Team, NASA Nexus for Exoplanet System Science.
}

of early T-type brown dwarfs demonstrated that the cloud thickness is constantly modulated by planetary-scale waves (Apai et al. 2017), and that continuously evolving light curves are very common in brown dwarfs. The large-scale waves are possibly caused by feedbacks between the cloud layer and atmospheric dynamics: one-dimensional models found oscillations caused by latent heat (Tan \& Showman 2017), and threedimensional models found small- and large-scale waves and oscillations in coupled atmospheric dynamics and cloud evolution systems (Showman et al. 2018). The picture that emerged for these $\mathrm{T}$ dwarfs is probably valid for most $\mathrm{T}$ dwarfs: Metchev et al. (2015) found that $36_{-17}^{+26} \%$ of the T dwarfs in their 16 object sample observed with Spitzer at 3.6 and $4.5 \mu \mathrm{m}$ are variable with amplitudes between $0.8 \%$ and $4.6 \%$. This finding indicates that clouds are typical to T-dwarf photospheres.

Similarly, photometric variability probably due to heterogeneous cloud coverage has been found in unbound late- $\mathrm{L}$ and $\mathrm{T}$ planetary-mass objects and companions to stars. Biller et al. (2015) found $J$-band high-amplitude photometric variability in PSO J318.522, an L7 dwarf with an estimated mass of $\sim 8 M_{\text {Jup. }}$. In addition, Biller et al. (2018) reported phase shifts between the Spitzer InfraRed Array Camera (IRAC) light curve and those derived from the near-infrared Hubble Space Telescope (HST), Wide Field Camera 3 (WFC3) spectra. Other 
examples of photometric or spectrophotometric variability found for planetary-mass objects include GU Psc b, a 9-13 $M_{\text {Jup }}$ T3.5 companion with $\sim 4 \%$ of variability amplitude (Naud et al. 2017); 2M1207b, a 2.3-4.8 $M_{\text {Jup }}$ L5 companion, with variability amplitude up to $1.36 \%$ (Zhou et al. 2016); and 2MASS J11193254-1137466AB and WISEA J114724.10204021.3, two L7 dwarfs with masses of 4-6 $M_{\text {Jup }}$, and variability amplitudes up to $\sim 2 \%$ and $\sim 1 \%$ in the [3.6] and [4.5] Spitzer channels, respectively (Schneider et al. 2018). Furthermore, two of the brown dwarfs (SIMP0136 and 2MASS J13243553+6358281) for which Apai et al. (2017) reported planetary-scale wave-modulated cloud thickness variations, are also likely in the planetary-mass range (Gagné et al. 2017, 2018).

Small amplitude rotational modulations appear also to be common in Y dwarfs. Cushing et al. (2016) has discovered photometric variability in WISE J140518.39+553421.3, a Y0.5 brown dwarf with an estimated mass between 9 and $21 M_{\text {Jup }}$ (Leggett et al. 2017). Cushing et al. (2016) measured a variability amplitude up to $3.5 \%$ in the [3.6] and [4.5] Spitzer channels. They found that the amplitude was different in two epochs, leading them to conclude that the cloud structures might evolve with time. Leggett et al. (2016) has also detected photometric variations in another Y0 dwarf (WISEP $\mathrm{J} 173835.52+273258.9$ ) in the Spitzer/IRAC bands and also identified likely near-infrared variability. Finally, Esplin et al. (2016) found up to 5\% variability on the coldest know brown dwarf WISE J085510.83-071442.5 (Y2) at the [3.6] and [4.5] Spitzer bands.

In conclusion, these examples show that clouds with varying vertical structures are also present in the coolest and lowest mass brown dwarfs and planetary-mass objects; but most of our knowledge currently is derived from $\mathrm{L} / \mathrm{T}$ transition objects, which are relatively bright and most likely to have highamplitude modulations (Radigan et al. 2014).

For Ross 458C, Burgasser et al. (2010), Burningham et al. (2011), and Morley et al. (2012) suggested that it should have a cloudy atmosphere as well. Nevertheless, Metchev et al. (2015) observed Ross 458C with the Spitzer [3.6] and [4.5] channels during a 21-hour-long continuous observation, concluding that its variability was not higher than $1 \%$ at those wavelengths. In this work, we show the existence of spectrophotometric variability in the very late T-spectral type Ross $458 \mathrm{C}$ in timeresolved spectra acquired with the WFC3 on board the HST during seven consecutive spacecraft orbits.

\section{Ross 458C}

Ross 458C (R.A. 13:00:41.15, decl. +12:21:14.22) is a T8 spectral type brown dwarf, with $J_{\text {UKIDSS }}=16.69 \pm 0.01$, and anomalously red in the near-infrared $(J-K=-0.21 \pm 0.06)$. Ross 458C was discovered as a companion to a high proper motion binary system, Ross 458AB, in the Data Release 5+ of the UKIRT Deep Infrared Sky Survey (UKIDSS) Large Area Survey (Goldman et al. 2010; Scholz 2010). Ross 458AB is a M0.5/M7.0 binary system at $11.51 \pm 0.02$ pc (Gaia Collaboration et al. 2018). Ross 458A is very active, and shows strong $\mathrm{H} \alpha$ emission and photometric variability (Hawley et al. 1997), indicating a likely maximum age of the system of 400-800 Myr (West et al. 2008).

Burgasser et al. (2010) used a $V-K / M_{K}$ color-magnitude diagram to estimate the metallicity of Ross 458A (Johnson \& Apps 2009; Schlaufman \& Laughlin 2010), and thus the metallicity of the system. They obtained a metallicity between $[\mathrm{Fe} / \mathrm{H}]=+0.31 \pm 0.05$ and $[\mathrm{Fe} / \mathrm{H}]=+0.20 \pm 0.05$ for the metallicity calibrations of Johnson \& Apps (2009) and Schlaufman \& Laughlin (2010), respectively. Both calibrations, thus, argue for supersolar metallicity. Burgasser et al. (2010) did not find $\mathrm{Li}$ absorption at $6708 \AA$ in the red optical spectrum of Ross 458A, setting 30-50 Myr as a lower limit for the age of the system. Consistent with this estimate, the equivalent widths of the alkali lines in Ross 458AB are larger than those of stars of similar spectral types in the Pleiades open cluster (112 \pm 5 Myr; Dahm 2015), indicating that Ross 458AB is older than Pleiades. At the same time, Ross 458A and B are not tidally locked (West et al. 2008), implying an age lower than $\sim 1$ Gyr. The BANYAN $\Sigma$ tool (Gagné et al. 2018), which estimates the probability of membership to young moving groups using the kinematics of the targets, provides a $99.3 \%$ probability for the Ross 458ABC system belonging to the Carina Near Moving Group, with an estimated age of $200 \pm 50$ Myr (Zuckerman et al. 2006).

\section{Observations and Data Reduction}

Ross 458C was observed in Cycle 23 of the HST program (PI: D. Apai, GO-14241) using the WFC3 in its near-infrared channel and G141 grism (MacKenty et al. 2010). The WFC3/ G141 grism covers the wavelength range between 1.05 and $1.70 \mu \mathrm{m}$, with a spectral resolving power of 130 at $1.4 \mu \mathrm{m}$. WFC3/IR has an image scale of 0 !" $13 /$ pixel.

We acquired seven consecutive orbits of observation on 2018 January 6 . In each $\sim 95$-minute-long orbit during the uninterrupted target visibility periods we obtained $11 \mathrm{G} 141$ frames with an integration time of $201.4 \mathrm{~s}$ each. To obtain an accurate wavelength reference for wavelength calibration, four direct (spectrally nondispersed) images in each orbit were also taken in the F132N filter. We used a $256 \times 256$ subarray mode to eliminate intra-orbit dead-time due to readout overheads. We performed the data reduction using the same method as in previous works published by our group (e.g., Apai et al. 2013; Buenzli et al. 2014, 2015; Lew et al. 2016; Manjavacas et al. 2018; Zhou et al. 2018).

We restricted the spectral range studied to $1.20-1.32$ and $1.54-1.60 \mu \mathrm{m}$ to avoid the noise at the edges of the spectra (due to the drop in the instrument sensitivity) and in the $1.40 \mu \mathrm{m}$ water band (where the source flux is very low). After reduction the average spectrophotometric uncertainty in measured intensity per spectral bin is $0.34 \%$. The measured uncertainties are due to photon and read noise, plus potential residual systematic errors of recognized origins that we discuss in Section 4.

\section{Systematics Assessment}

\subsection{Ramp Effect Correction}

The most prominent systematics in WFC3 near-infrared time-resolved photometry is the ramp effect. We corrected this systematic effect using the process described in Zhou et al. (2017) for which models charge trapping and delayed release in the detector. This reproduced the ramp effect with very high fidelity, allowing us to correct for it. 


\subsection{Pointing Stability}

High-precision differential spectrophotometry requires subpixel line-of-sight pointing stability. Such performance is usually delivered by the HST pointing control system but, infrequently, anomalously high pointing drifts can arise. We evaluated, and confirmed, the requisite level of pointing stability during our Ross $458 \mathrm{C}$ observations as follows for each of the 77 spectra taken during the seven $H S T$ orbits. We measured the positions the photocentric peaks of the spectra in the cross-dispersion direction by Gaussian profile fitting at six equally separated wavelength locations along the spectra. We found that the dispersion in Gaussian-fit peaks in the image ensemble was at most $10 \%$ of a pixel (13 mas). This is the nominal value for the observations and it is much smaller than the aperture size (4 pixels) that we used to extract the spectra. Thus, we conclude that the pointing instability does not affect our measurements significantly.

\subsection{Sky Background Variations}

Ross $458 \mathrm{C}$ is a companion to the Ross $458 \mathrm{AB}$ binary system with an AB-C separation of $102^{\prime \prime}$. This separation is much larger that the field of the WFC3 near-infrared detector $\left(33^{\prime \prime} \times 33^{\prime \prime}\right.$ in the $256 \times 256$ subarray), therefore, we do not expect the flux of the binary to contaminate the flux of Ross 458C. Nevertheless, we tested if the flux of the sky (after sky subtraction) is correlated with the spectral-photometric variability found in the light curve of Ross 458C. We measured the flux of the sky as a function of time in one rectangular aperture of $146 \times 8$ pixels above $(x=447-593$, $y=552-560)$ and one below $(x=447-593 \quad y=513-521)$ the spectral trace.

We calculated the $\tau$ Kendall's coefficient and its significance to quantitatively test for a correlation between sky background measurements in the two regions and the variability measurements of the white light curve $(1.10-1.60 \mu \mathrm{m})$ in the 77 images using the r_correlate.pro IDL function. For the sky measured in the upper region of the Ross $458 \mathrm{C}$ spectra, we obtained a $\tau=-0.08$ with a significance of 0.33 , and for the region below the target's spectra we found a correlation of $\tau=-0.02$, with a significance of 0.75 . Both $\tau$ values are close to zero, indicating no correlation between the target's spectrophotometric variability and the sky background.

\section{Results}

In Figure 2 we present the white, narrow $J$-band (JNB, $1.21-1.32 \mu \mathrm{m}$ ) and narrow $H$-band light curves (HNB, $1.54-1.60 \mu \mathrm{m})$ for Ross $458 \mathrm{C}$ after charge trapping correction. All three light curves show quasi-periodic variations.

\subsection{Spectral Variability}

Following Apai et al. (2013), we study the amplitude of the rotational modulations as a function of wavelength by comparing the average of the three maximum and three minimum spectra among the 77 spectra taken (see Figure 1, top panel). In the bottom panel of Figure 1 we show the ratio between the three brightest spectra and the three faintest spectra -in other words, the relative amplitude across the spectral wavelength range.

The relative peak-to-peak signal of the rotational modulation for Ross $458 \mathrm{C}$ is $2.62 \pm 0.02 \%$ as measured in the white and


Figure 1. Top panel: average of the three maximum (purple color) and the three minimum (black color) spectra of the 77 spectra taken during seven $H S T$ orbits for Ross 458C. Middle panel: ratio between the three spectra with the maximum flux and three spectra with the minimum flux of Ross $458 \mathrm{C}$. We found a best linear fit to the $J$ - and $H$-band ratios to show the color trend found in both bands. Bottom panel: residuals of the ratio between the three maximum and minimum spectra after subtracting the best linear fit for both bands.

in the JNB light curve. We measured a marginal rotational modulation of $3.16 \pm 1.36 \%(2.2 \sigma)$ in the HNB light curve. Because in several wavelength ranges of the spectra the flux density is close to zero $(1.10-1.20 \mu \mathrm{m}, 1.32-1.43 \mu \mathrm{m}$, and $1.63-1.69 \mu \mathrm{m}$ ), the ratio of these flux densities is very noisy. Therefore, we cannot derive any conclusion about the amplitude of the variations inside and outside the water band $(1.35-1.43 \mu \mathrm{m})$. Outside the wavelength ranges (with a signalto-noise bigger than 20), a color trend (larger amplitude variations at shorter wavelengths) is visible in the narrow $J$ band, as shown by the best linear fit (Figure 1, middle panel), and also to some extent in the $H$-band, although the latter color trend is less significant (Figure 1, middle panel). In the bottom panel of Figure 1 we show the residuals after the subtraction of the best linear fit to the ratio of the three maximum and minimum spectra.

\subsection{Rotational Period Estimates}

We aim to estimate the rotational period of Ross $458 \mathrm{C}$ using its white light curve acquired in seven consecutive $H S T$ orbits, with an end-to-end duration of approximately $10 \mathrm{hr}$. We use three different methods to estimate the tentative rotational period of Ross $458 \mathrm{C}$. We assume that the quasi-periodic modulations we detected in its light curve are a good initial measure of the rotation period (see Apai et al. 2017). In addition, we assume that the variability is due to rotational modulations of a hemisphere-integrated signal emerging from an atmosphere with heterogeneous cloud cover, which is the most plausible possibility and consistent with the evidence 
Table 1

Parameters for the Sine Function Fit

\begin{tabular}{|c|c|c|c|}
\hline Sine Component & White Fit & JNB-band Fit & HNB-band Fit \\
\hline Period (hr) & $6.59 \pm 0.21$ & $6.86 \pm 0.30$ & $5.62 \pm 0.38$ \\
\hline$A_{0}$ & $1.00 \pm 0.01$ & $1.00 \pm 0.01$ & $1.00 \pm 0.01$ \\
\hline$A_{1}$ & {$[-7.06 \pm 0.52] \times 10^{-3}$} & {$[-7.06 \pm 0.74] \times 10^{-3}$} & {$[-7.03 \pm 1.56] \times 10^{-3}$} \\
\hline$\chi^{2}$ & 93.1 & 45.0 & 17.9 \\
\hline Number data points & 77 & 77 & 77 \\
\hline
\end{tabular}

presented in the literature (see, for example, the discussion in Section 5 of Manjavacas et al. 2018).

We estimate the rotational period of ROSS 458C using the three following methods.

1. Sine function fitting. We fit a sine function to the all three light curves. We chose a sine wave as it is the simplest description of the light curve, but we note that the actual light curve might be more complex. We fit a sine function using the following expression to each of these three spectral regions:

$$
F(t)=A_{0}+A_{1} \sin \left[\frac{2 \pi t}{P}+\phi\right],
$$

where $A_{0}$ is the base level of the light curve, $A_{1}$ is the amplitude $t$ is the time, $P$ is the period of the sine wave, and $\phi$ its phase.

We performed a Levenberg-Marquardt least-squares fit to the sine function using the mpfitfun.pro IDL function (Markwardt 2009). This IDL function provides an estimate of the uncertainties using the uncertainties of the light curve data points, and by minimizing the value of the $\chi^{2}$. We obtained consistent periods for the white light curve $(6.59 \pm 0.21 \mathrm{hr})$ and the narrow $J$-band light curve $(6.86 \pm 0.30 \mathrm{hr})$, but not for the narrow $H$-band light curve $(5.62 \pm 0.38 \mathrm{hr})$. We obtained consistent phases for the white $(1.40 \pm 0.18 \mathrm{rad})$ and narrow $J$-band light curve (1.65 $\pm 0.23 \mathrm{rad})$, but a marginally significant different phase $(2.5 \sigma)$ for the narrow $H$-band light curve $(0.44 \pm 0.42 \mathrm{rad})$. Thus, there is an indication of a phase shift between the $J$ - and the $H$-narrow bands, at a $2.4 \sigma$ confidence level. The residuals after the subtraction of the best-fit sine wave follow a Gaussian distribution for the white and $J$-band light curves, but not for the narrow $H$ band light curve. This indicates that there might be underlying (non-sinusoidal) structures in the narrow $H$ band light curve. In fact, under the assumption of the light curve being a sine wave with the same period in both bands, there is no evidence for a phase shift, with phases of $1.66 \pm 0.12 \mathrm{rad}$ for the JNB light curve and $1.73 \pm 0.26$ rad for HNB light curve.

Table 1 lists the parameters of the best-fit sine functions for the white, $J$-band, and narrow $H$-band light curves. In the top panels of Figure 2 we show the best-fit sine functions for the white, narrow $J$-band, and $H$ - band light curves. In the middle panels we show the light curve residuals after the subtraction of the best-fit sine functions. The bottom panels show the white, narrow $J$ band (JNB), and $H$-band (HNB) phase-folded light curves.

2. Bayesian Generalized Lomb-Scargle Periodogram (BGLS). As discussed in Manjavacas et al. (2018), the regular Lomb-Scargle periodogram (Horne \& Baliunas 1986) does not consider data point uncertainties. It has been shown that this periodogram analysis provides biased results in the case of temporal gaps in the data, such as in the case of the HST data (Mortier et al. 2015; Cowan et al. 2017). To solve this problem, Mortier et al. (2015) presented a Python-based code to calculate the BGLS periodogram of a time-series based on the algorithms presented in Bretthorst (2001) and Zechmeister \& Kürster (2009). These consider uncertainties of the data points, data gaps, allow for zero-point differences in data collected at different epochs, and also provide the probabilities of peaks of similar power in the conventional Lomb-Scargle Periodogram. With this approach we obtained a period of $6.60 \pm 0.89 \mathrm{hr}$ for the white light curve, a period of $6.86 \pm 0.86 \mathrm{hr}$ for the narrow $J$-band light curve, and a period of $5.63 \pm 1.37 \mathrm{hr}$ for the narrow $H$-band light curve. Uncertainties are computed as the FWHM of a Gaussian function fitted to the peaks.

3. Monte Carlo simulation. As a third approach we used a Monte Carlo method as in Manjavacas et al. (2018) to robustly estimate the periods and their uncertainties. We created 1000 synthetic light curves based on the observed white, JNB, and HNB light curves, and shifted each data point using normally distributed random values, using the mean of the Gaussian as the measured flux and its standard deviation as its uncertainty. We produced the traditional Lomb-Scargle periodogram of each simulated light curve. We calculated the period as the peak of the $50 \%$ percentile curve and its uncertainty as the FWHM of that percentile as in Manjavacas et al. (2018). We obtained a period of $6.75 \pm 1.58 \mathrm{hr}$ for the white light curve, a period of $6.91 \pm 1.51 \mathrm{hr}$ for the narrow $J$-band light curve, and a $6.27 \pm 1.55 \mathrm{hr}$ period for the narrow $H$ band light curve, in agreement with the periods obtained using the previous methods. In this case, we obtained the regular Lomb-Scargle periodogram because the power of the peaks obtained are normalized to the variance of the data, which is similar for each generated light curve.

As explained above, the light curve might not be, in fact, a sine wave but a more complex function (consistent with highquality light curves obtained for other objects). However, our current data do not warrant more complex models. Thus, we decided to adopt as a likely period, the one obtained in the Monte Carlo simulation, $6.75 \pm 1.58 \mathrm{hr}$, with its more conservative uncertainty. 

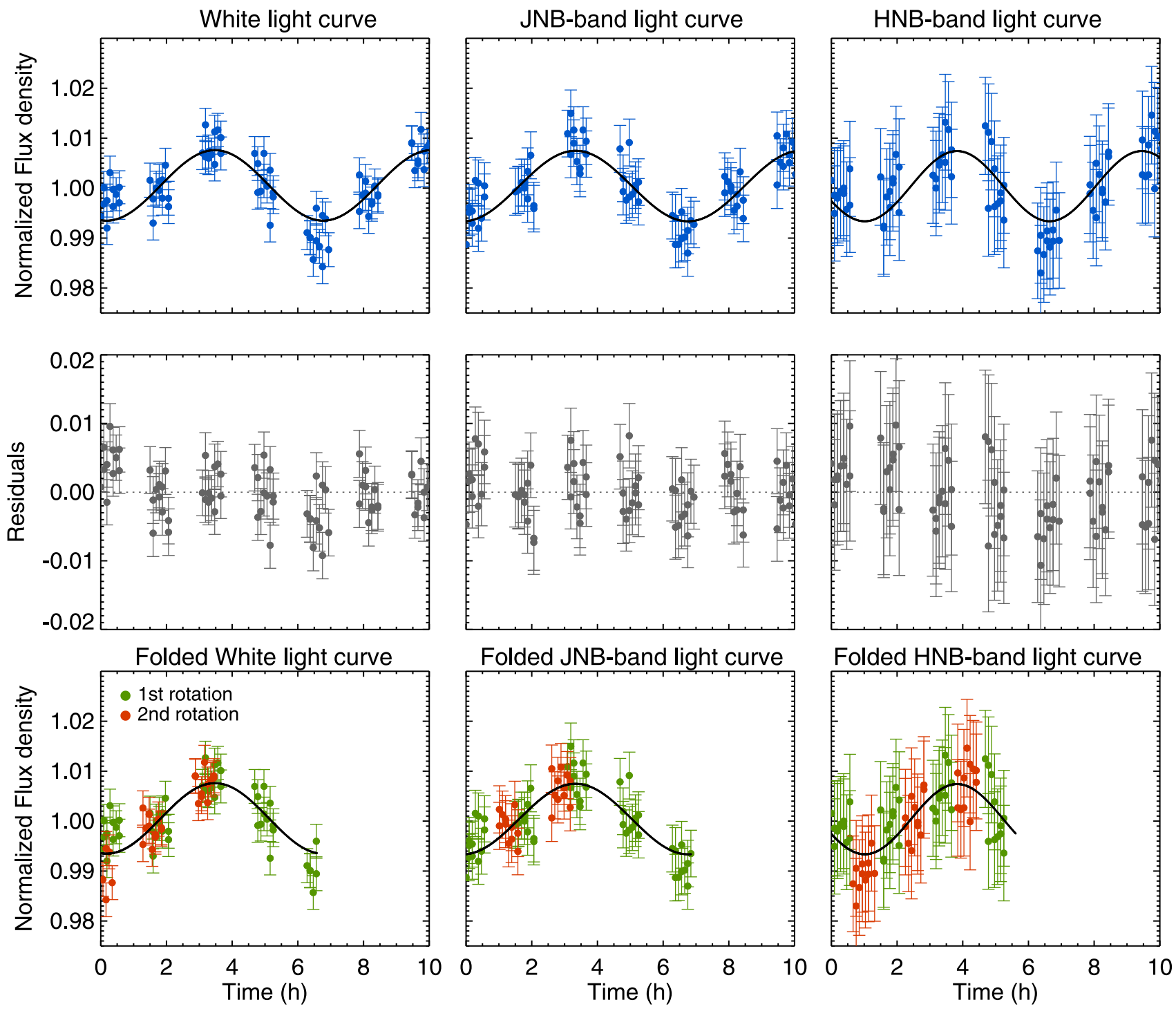

Figure 2. Upper panels show the best-fit sine function to the white, narrow $J$-band, and the narrow $H$-band light curves. The middle panels show the residuals after subtracting the best-fit sine wave from the white, $J$-band, and narrow $H$-band light curves. The bottom panels show the respective phase-folded light curves

\section{Discussion \\ 6.1. Sulfide Clouds in Ross 458 C}

Burgasser et al. (2010) and Burningham et al. (2011) compared the near-infrared Ross $458 \mathrm{C}$ spectra taken with the Folded-port Infrared Echelle instrument at Mallegan, and the spectra obtained with the Infrared Camera and Spectrograph at Subaru, and its mid-infrared IRAC photometry to several cloudless and cloudy atmospheric models from Saumon \& Marley (2008) and from Allard et al. (2001; BT-Settl atmospheric models). Both studies concluded that the cloudy BT-Settl models provide a significantly better match to the data than the cloud-free atmosphere models.

Morley et al. (2012) generated new atmospheric models based on the Ackerman \& Marley (2001) cloud models for brown dwarfs and planetary-mass objects of temperatures between 400 and $1200 \mathrm{~K}, \log g=4.0-5.5$, and condensate efficiencies, $f_{\text {eff }}$, from 2 to 5 . In agreement with Burgasser et al. (2010) and Burningham et al. (2011), they concluded that cloudy atmospheric models matched the near-infrared spectrum of Ross 458C significantly better than cloud-free models.
Clouds would also explain the red color of Ross 458C. Morley et al. (2012) proposed that the emergence of the sulfide clouds, specifically $\mathrm{Na}_{2} \mathrm{~S}$, might be a more natural explanation for the cloudy atmospheres predicted for Ross $458 \mathrm{C}$, than the reemergence of silicate clouds, as Burgasser et al. (2010) and Burningham et al. (2011) proposed.

The discovery of rotational modulations in the near-infrared $H S T$ /WFC3 spectra in our seven HST orbits also points to the existence of clouds in the late-T Ross 458C. This finding is consistent with the conclusions of Morley et al. (2012), that predicted the presence clouds of sulfide composition. Our observations of near-infrared modulations in Ross 458C extends temporally resolved spectral studies of clouds toward the very end of the $\mathrm{T}$ spectral type sequence. The detected modulations are inconclusive in the HNB, but display a strongly wavelength-dependent slope in the $J$-band that has not yet been described in the literature. If confirmed, this colordependent amplitude may suggest differences in grain size distributions between silicate clouds (probed in early T dwarfs) and sulfide clouds probably observed in the late $\mathrm{T}$ and $\mathrm{Y}$ dwarfs. The presence of the rotational modulations in a T8 


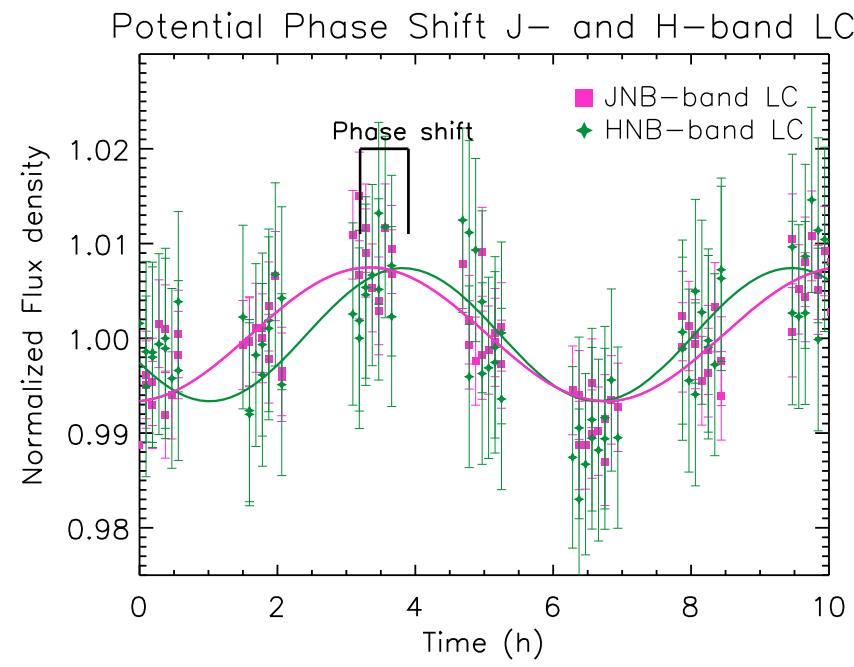

Figure 3. Narrow $J$ - (pink points) and $H$-band (green points) light curves with their respective best fitting sine waves color coded with the same colors as their respective light curves.

dwarf itself suggests that clouds are typical to most ultra-cool atmospheres, although the composition and structure of the clouds is likely to differ.

\subsection{Comparison with 2MASS 22282889-4310262}

Both 2MASS 22282889-4310262 and Ross 458C show quasi-sinusoidal light curves, but with different rotational periods of $\sim 1.42 \mathrm{hr}$ (Buenzli et al. 2012) and $\sim 6.75 \mathrm{hr}$, respectively. Both JNB and HNB light curves show phase shifts of $1.65 \pm 0.23 \mathrm{rad}$ and $0.44 \pm 0.42 \mathrm{rad}$ that correspond to $1.80 \pm 0.25 \mathrm{hr}$ and $0.39 \pm 0.38 \mathrm{hr}$. The likely phase shift is $1.41 \pm 0.45 \mathrm{hr}$, as shown in Figure 3, when measured at the peak of the first light curve). In contrast, for 2MASS 22282889-4310262 Buenzli et al. (2012) found that the phase shifts correlated with the pressure, which was interpreted as evidence for large-scale, longitudinal-vertical structures. Several years later, follow-up observations by Yang et al. (2016) demonstrate that the phase shifts were still present, arguing for either long-lived or frequently occurring longitudinal-vertical cloud structures. If a similar phase shift is confirmed in Ross $458 \mathrm{C}$, it would suggest that such vertical-longitudinal structures are not rare in late T-type brown dwarfs. Buenzli et al. (2012) measured a phase shift of $15^{\circ} \pm 2^{\circ}(0.26 \pm 0.03 \mathrm{rad})$ for 2MASS 22282889-4310262 between the JNB and the HNB. In addition, in their two visits Yang et al. (2016) have measured phase shifts of $5^{\circ} \pm 2^{\circ}(-0.08 \pm 0.03 \mathrm{rad})$ and $8^{\circ} \pm 2^{\circ}(-0.14 \pm 0.03 \mathrm{rad})$. Finally, Ross $458 \mathrm{C}$ has a rotational modulation amplitude of $2.62 \pm 0.02 \%$, and $2 \mathrm{MASS}$ 22282889-4310262 has a rotational modulation amplitude between $1.45 \%$ and $5.30 \%$ as measured by Buenzli et al. (2012).

Buenzli et al. (2012) fitted several atmospheric models to the spectrum of 2M2228, finding a best match with the $T_{\text {eff }}=900 \mathrm{~K}, \log g=4.5, f_{\text {sed }}=3$ for the Marley et al. (2012) models with sulfide clouds. Similarity, Morley et al. (2012) found a best match with sulfide clouds atmospheric models of $T_{\text {eff }}=700 \mathrm{~K}, \log g=4.0, f_{\text {sed }}=3$ for Ross $458 \mathrm{C}$. These two examples support the prediction by Morley et al. (2012) that sulfide clouds layer with patchy clouds that condensate at temperatures cooler than $900 \mathrm{~K}$ could be found in late-T brown dwarfs/planetary-mass objects, and be the cause of their spectrophotometric variability.

\section{Conclusions}

1. We discovered rotational modulations in the spectrum of the planetary-mass object Ross $458 \mathrm{C}$. This source is the latest spectral type object for which spectral variability has been found.

2. We detected rotational modulations with a peak-to-peak signal of $2.62 \pm 0.02 \%$ over the entire $1.1-1.64 \mu \mathrm{m}$ wavelength range.

3. Considering the results given by the method that provides the most conservative uncertainties, we find very similar rotational periods for the white $(6.75 \pm 1.58 \mathrm{hr})$ and JNB light curves $(6.91 \pm 1.51 \mathrm{hr})$, and for the HNB light curve $(6.27 \pm 1.55 \mathrm{hr})$.

4. Allowing different periods in the JNB and HNB light curves, we found a potential $(2.5 \sigma$ level $)$ phase shift between the JNB $(1.65 \pm 0.23 \mathrm{rad})$ and the HNB light curves $(0.44 \pm 0.42 \mathrm{rad}), \quad$ corresponding to $1.41 \pm 0.45 \mathrm{hr}$ when measured at the peak of the first light curve). A phase shift has also been detected in the T6.5 brown dwarf 2M2228 (Buenzli et al. 2012; Yang et al. 2015). However, under the assumption that the light curve is a sine wave with the same period in both bands, there is no evidence for a phase shift. Follow-up observations are required to confirm this tentative phase shift in Ross 458C.

5. The ratio of the three maximum and the three minimum spectra across the 1.1-1.64 $\mu \mathrm{m}$ wavelength range shows a color trend-not seen previously-in the narrow $J$ band. However, the modulations are gray in the $H$ narrow band.

The detection of rotational modulations in Ross $458 \mathrm{C}$ extends detailed cloud studies to the coolest $\mathrm{T}$ dwarfs (T8), offering an opportunity for follow-up observations to study clouds potentially composed of sulfides. Furthermore, Ross $458 \mathrm{C}$ further increases the small group of directly imaged planetary-mass companions, where cloud properties and rotational modulations can be studied.

Based on observations made with the NASA/ESA Hubble Space Telescope, obtained at the Space Telescope Institute, which is operated by AURA, Inc., under NASA contract NAS 5-26555, under GO-14241. This publication makes use of data products from the Two Micron All Sky Survey, which is a joint project of the University of Massachusetts and the Infrared Processing and Analysis Center/California Institute of Technology, funded by the National Aeronautics and Space Administration and the National Science Foundation.

\section{ORCID iDs}

Elena Manjavacas (iD https://orcid.org/0000-0003-0192-6887

Dániel Apai (1) https://orcid.org/0000-0003-3714-5855

Ben W. P. Lew (1) https://orcid.org/0000-0003-1487-6452

Yifan Zhou (ib https://orcid.org/0000-0003-2969-6040

Glenn Schneider (iD https://orcid.org/0000-0002-4511-5966

Adam J. Burgasser (iD https://orcid.org/0000-0002-6523-9536

Theodora Karalidi (i) https://orcid.org/0000-0001-7356-6652

Paulo A. Miles-Páez (iD https://orcid.org/0000-0003-

2446-8882 
Patrick J. Lowrance (1) https://orcid.org/0000-0001-8014-0270

Nicolas Cowan (1) https://orcid.org/0000-0001-6129-5699

Mark S. Marley (1) https://orcid.org/0000-0002-5251-2943

Stan Metchev (1) https://orcid.org/0000-0003-3050-8203

\section{References}

Ackerman, A. S., \& Marley, M. S. 2001, ApJ, 556, 872

Allard, F., Hauschildt, P. H., Alexander, D. R., Tamanai, A., \& Schweitzer, A. 2001, ApJ, 556, 357

Apai, D., Karalidi, T., Marley, M. S., et al. 2017, Sci, 357, 683

Apai, D., Radigan, J., Buenzli, E., et al. 2013, ApJ, 768, 121

Artigau, É., Bouchard, S., Doyon, R., \& Lafrenière, D. 2009, ApJ, 701, 1534

Biller, B. A., Vos, J., Bonavita, M., et al. 2015, ApJL, 813, L23

Biller, B. A., Vos, J., Buenzli, E., et al. 2018, AJ, 155, 95

Bretthorst, G. L. 2001, in AIP Conf. Ser. 568, Bayesian Inference and Maximum Entropy Methods in Science and Engineering, ed. A. Mohammad-Djafari (Melville, NY: AIP), 246

Buenzli, E., Apai, D., Morley, C. V., et al. 2012, ApJL, 760, L31

Buenzli, E., Apai, D., Radigan, J., Reid, I. N., \& Flateau, D. 2014, ApJ, 782, 77

Buenzli, E., Saumon, D., Marley, M. S., et al. 2015, ApJ, 798, 127

Burgasser, A. J., Kirkpatrick, J. D., Brown, M. E., et al. 2002a, ApJ, 564, 421

Burgasser, A. J., Marley, M. S., Ackerman, A. S., et al. 2002b, ApJL, 571, L151

Burgasser, A. J., Simcoe, R. A., Bochanski, J. J., et al. 2010, ApJ, 725, 1405

Burningham, B., Leggett, S. K., Homeier, D., et al. 2011, MNRAS, 414, 3590

Cowan, N. B., Chayes, V., Bouffard, É., Meynig, M., \& Haggard, H. M. 2017, MNRAS, 467, 747

Cushing, M. C., Hardegree-Ullman, K. K., Trucks, J. L., et al. 2016, ApJ, 823,152

Cushing, M. C., Rayner, J. T., \& Vacca, W. D. 2005, ApJ, 623, 1115

Dahm, S. E. 2015, ApJ, 813, 108

Esplin, T. L., Luhman, K. L., Cushing, M. C., et al. 2016, ApJ, 832, 58

Gagné, J., Allers, K. N., Theissen, C. A., et al. 2018, ApJL, 854, L27

Gagné, J., Faherty, J. K., Burgasser, A. J., et al. 2017, ApJL, 841, L1

Gaia Collaboration, Brown, A. G. A., Vallenari, A., et al. 2018, A\&A, 616, 1

Goldman, B., Marsat, S., Henning, T., Clemens, C., \& Greiner, J. 2010, MNRAS, 405, 1140

Hawley, S. L., Gizis, J. E., \& Reid, N. I. 1997, AJ, 113, 1458
Horne, J. H., \& Baliunas, S. L. 1986, ApJ, 302, 757

Johnson, J. A., \& Apps, K. 2009, ApJ, 699, 933

Kirkpatrick, J. D. 2005, ARA\&A, 43, 195

Leggett, S. K., Cushing, M. C., Hardegree-Ullman, K. K., et al. 2016, ApJ, 830, 141

Leggett, S. K., Tremblin, P., Esplin, T. L., Luhman, K. L., \& Morley, C. V. 2017, ApJ, 842, 118

Lew, B. W. P., Apai, D., Zhou, Y., et al. 2016, ApJL, 829, L32

MacKenty, J. W., Kimble, R. A., O'Connell, R. W., \& Townsend, J. A. 2010, Proc. SPIE, 7731, 77310Z

Manjavacas, E., Apai, D., Zhou, Y., et al. 2018, AJ, 155, 11

Markwardt, C. B. 2009, in ASP Conf. Ser. 411, Astronomical Data Analysis Software and Systems XVIII, ed. D. A. Bohlender, D. Durand, \& P. Dowler (San Francisco, CA: ASP), 251

Marley, M. S., Saumon, D., Cushing, M., et al. 2012, ApJ, 754, 135

Metchev, S. A., Heinze, A., Apai, D., et al. 2015, ApJ, 799, 154

Morley, C. V., Fortney, J. J., Marley, M. S., et al. 2012, ApJ, 756, 172

Mortier, A., Faria, J. P., Correia, C. M., Santerne, A., \& Santos, N. C. 2015 A\&A, 573, A101

Naud, M.-E., Artigau, É., Rowe, J. F., et al. 2017, AJ, 154, 138

Radigan, J., Jayawardhana, R., Lafrenière, D., et al. 2012, ApJ, 750, 105

Radigan, J., Lafrenière, D., Jayawardhana, R., \& Artigau, E. 2014, ApJ, 793, 75

Saumon, D., \& Marley, M. S. 2008, ApJ, 689, 1327

Schlaufman, K. C., \& Laughlin, G. 2010, A\&A, 519, A105

Schneider, A. C., Hardegree-Ullman, K. K., Cushing, M. C., Kirkpatrick, J. D., \& Shkolnik, E. L. 2018, AJ, 155, 238

Scholz, R.-D. 2010, A\&A, 515, A92

Showman, A. P., Tan, X., \& Zhang, X. 2018, arXiv:1807.08433

Tan, X., \& Showman, A. P. 2017, ApJ, 835, 186

West, A. A., Hawley, S. L., Bochanski, J. J., et al. 2008, AJ, 135, 785

Yang, H., Apai, D., Marley, M. S., et al. 2015, ApJL, 798, L13

Yang, H., Apai, D., Marley, M. S., et al. 2016, ApJ, 826, 8

Zechmeister, M., \& Kürster, M. 2009, A\&A, 496, 577

Zhou, Y., Apai, D., Lew, B. W. P., \& Schneider, G. 2017, AJ, 153, 243

Zhou, Y., Apai, D., Metchev, S., et al. 2018, AJ, 155, 132

Zhou, Y., Apai, D., Schneider, G. H., Marley, M. S., \& Showman, A. P. 2016, ApJ, 818, 176

Zuckerman, B., Bessell, M. S., Song, I., \& Kim, S. 2006, ApJL, 649, L115 